\title{
Existence of the solutions to convolution equations with distributional kernels
}

\author{
Alexander G. Ramm $^{1 *}$ \\ ${ }^{1}$ Department of Mathematics, Kansas State University, Manhattan, KS 66506, USA \\ *Corresponding authorE-mail: ramm@math.ksu.edu
}

\begin{abstract}
It is proved that a class of convolution integral equations of the Volterra type has a global solution, that is, solutions defined for all $t \geq 0$. Smoothness of the solution is studied.

Keywords: Volterra equations; distributional kernels
\end{abstract}

\section{Introduction}

Consider the equation:

$u(t)=\int_{0}^{t} \frac{(t-s)^{a}}{\Gamma(a+1)} u(s) d s+f(t):=V u+f$,

where

$t \geq 0 ; \quad a=$ const $\neq-1,-2, \ldots$.

and

$V u:=V_{a} * u, \quad V_{a}:=\frac{t_{+}^{a}}{\Gamma(a+1)}$.

Here $\Gamma(z)$ is the Gamma function, and (3) is a convolution with the distribution $V_{a}$, see [1]. Thus, equation (1) is a Volterra equation with kernel that is not absolutely integrable for $a<-1$. There is a large literature on integral equations, [2], but the usual methods to study such equations are based on the assumption that the kernel of the operator $V$ belongs to $L^{p}$ with $p \geq 1$.

The goal of this paper is to develop a method to study (1) with a distributional kernel $\frac{t_{+}^{a}}{\Gamma(a+1)}$. The basic known result (see [1]) is the property of convolution

$V_{a} * V_{b}=V_{b} * V_{a}=V_{a+b}$.

Our result is formulated in Theorem 1 .

Theorem 1. Equation (1) with $a<-1$ is uniquely solvable. Its solution $u$ exists for all $t \geq 0$. It belongs to the space of functions which is of the same smoothness as $V_{-a} f$.

In the next section a proof is given.

\section{Proof}

Proof of Theorem 1 . The idea of the proof is to apply $V_{-a}$ to equation (1) and use the formula

$V_{a} * V_{-a}=I$, where $I$ is the identity operator whose kernel is the delta-function. Applying $V_{-a}$ to (1) one gets

$V_{-a} u=u+V_{-a} f$

or

$$
u=-V_{-a} u+V_{-a} f
$$

Suppose that $a<-1$. Then $-a>1$ and the operator $V_{-a}$ is a convolution with a continuous kernel. Therefore equation (7) is a Volterra integral equation with a continuous kernel. Consequently, this equation has a unique solution $u$ for all $t \geq 0$. This solution can be calculated by iterations. Equations (1) and (7) are equivalent because $V_{a} * V_{-a}=I$. Therefore, Theorem 1 is proved.

\section{References}

[1] I.Gelfand, G. Shilov, Generalized functions, Vol.1, AMS Chelsea Publ., 1964.

[2] P. Zabreiko, A.Koshelev, M. Krasnoselskii, S.Mikhlin, L. Rakovshchik, V Stecenko, Integral equations: a reference text, Leyden, Noordhoff International Publ., 1975. 